CIENCIAMATRIA

Revista Interdisciplinaria de Humanidades, Educación, Ciencia y Tecnología

Año VI. Vol. VI. N¹0. Enero - Julio 2020

Hecho el depósito de ley: pp201602FA4721

ISSN-L: 2542-3029; ISSN: 2610-802X

Universidad Nacional Experimental Francisco de Miranda (UNEFM). Santa Ana de Coro. Venezuela

Jorge Eduardo Jalil Ponce; Vicenta Elizabeth Pisco Sornoza; Johana María Zambrano Sornoza

DOI 10.35381/cm.v6i10.140

\title{
Competencias instrumentales del gerente en el sistema de Educacion Superior
}

\author{
Instrumental competences of the manager in the higher Education System
}

\author{
Jorge Eduardo Jalil Ponce \\ jejalil@hotmail.com \\ Universidad Técnica de Manabí \\ Ecuador \\ https://orcid.org/0000-0003-3631-1989 \\ Vicenta Elizabeth Pisco Sornoza \\ vpisco@utm.edu.ec \\ Universidad Técnica de Manabí \\ Ecuador \\ https://orcid.org/0000-0003-4399-0692 \\ Johana María Zambrano Sornoza \\ jmzambranos@utm.edu.ec \\ Universidad Técnica de Manabí \\ Ecuador \\ https://orcid.org/0000-0001-7264-1374
}

Recibido: 1 de mayo de 2019

Aprobado: 14 de junio 2019

\section{RESUMEN}

La consideración y ejecución de las competencias instrumentales en los gerentes universitarios permiten direccionar y controlar las acciones que se desarrollan bajo sus responsabilidades gerenciales. Bajo esta premisa, se consideró como objetivo identificar las competencias instrumentales del gerente en el sistema de educación superior. La metodología fue de tipo descriptiva con un diseño de campo, no experimental y transeccional. La técnica de investigación aplicada fue la encuesta medida a través de un cuestionario como instrumento de recolección de datos. Éste estuvo compuesto por 12 ítems con opciones de respuesta politómica que fue aplicado a los docentes con cargos gerenciales universitarios. Los datos aportados, permitieron posterior a su análisis 


\author{
CIENCIAMATRIA \\ Revista Interdisciplinaria de Humanidades, Educación, Ciencia y Tecnología \\ Año VI. Vol. VI. N¹0. Enero - Julio 2020 \\ Hecho el depósito de ley: pp201602FA4721 \\ ISSN-L: 2542-3029; ISSN: 2610-802X \\ Universidad Nacional Experimental Francisco de Miranda (UNEFM). Santa Ana de Coro. Venezuela \\ Jorge Eduardo Jalil Ponce; Vicenta Elizabeth Pisco Sornoza; Johana María Zambrano Sornoza
}

generar entre sus conclusiones que las competencias instrumentales que aplican los gerentes universitarios, se evaluaron en un nivel eficiente con relación a su cumplimiento.

Descriptores: Enseñanza superior; Enseñanza científica superior; Ciencias de la educación; Investigación pedagógica; Competencias del docente.

\begin{abstract}
The consideration and execution of instrumental competencies in university managers allow to direct and control the actions that are carried out under their management responsibilities. Under this premise, it was considered to be the objective of identifying the instrumental competencies of the manager in the higher education system. The methodology was descriptive with a field design, not experimental and transectional. The applied research technique was the survey measured through a questionnaire as a data collection tool. It consisted of 12 items with polytominomic response options that was applied to teachers with university management positions. The data provided, allowed after their analysis to generate among their conclusions that the instrumental competencies applied by university managers, were evaluated at an efficient level in relation to their compliance.
\end{abstract}

Descriptors: Higher education; Higher science education; Educational sciences; Educational research; Teacher qualifications.

\title{
INTRODUCCIÓN
}

Desde que las personas comenzaron a organizarse en grupos para alcanzar objetivos que no podían lograr individualmente, la gerencia ha sido fundamental para asegurar la coordinación de los esfuerzos colectivos. Al respecto, Melinkoff (2001) define la gerencia como: "la organización y dirección de recursos humanos y materiales para lograr los fines propuestos, tanto del sector público como del sector privado, mediante la utilización de un conjunto de procesos"(p.9).

En efecto, la gerencia se realiza a través del esfuerzo coordinado de los individuos para alcanzar los objetivos propuestos, con un mínimo de recursos y con el apoyo de las 


\section{CIENCIAMATRIA}

Revista Interdisciplinaria de Humanidades, Educación, Ciencia y Tecnología

Año VI. Vol. VI. N¹0. Enero - Julio 2020

Hecho el depósito de ley: pp201602FA4721

ISSN-L: 2542-3029; ISSN: 2610-802X

Universidad Nacional Experimental Francisco de Miranda (UNEFM). Santa Ana de Coro. Venezuela

Jorge Eduardo Jalil Ponce; Vicenta Elizabeth Pisco Sornoza; Johana María Zambrano Sornoza

personas, las cuales requieren de ciertas competencias para mantenerse en sus puestos de trabajo. De igual forma Terry (2005) define la gerencia como: "un proceso para lograr un objetivo predeterminado mediante el esfuerzo humano y la utilización de un conjunto de procesos"(p.70). En este orden, la gerencia requiere de ciertas competencias instrumentales y valores para coordinar los recursos técnicos y humanos hacia la intención de la organización, la tarea esencial es integrar y coordinar los recursos, tales como personas, materiales y financieros, para alcanzar los objetivos de manera eficaz.

Las competencias gerenciales en el nivel de educación superior pueden ser explicadas por una serie de teorías administrativas, sin embargo todas coinciden en que las competencias se vinculan con los conocimientos prácticos y los valores que orientan sus funciones. Al respecto, Haberman (2008) dentro de la teoría crítica, vincula la acción con las competencias al explicar "la relación pensamiento, acción, mediante la construcción y reconstrucción del saber humano, a través de tres intereses, entre éstos: el interés técnico, el interés práctico y el emancipador, que llamó "intereses constitutivos del saber"(p.36).

En este orden, se entienden como las acciones conformadas por eventos validados intersubjetivamente. Es un valor referente al intento de generar consecuencias intencionadas. Las competencias convierten a un sujeto en capaz de coordinar lenguaje y acción, así mismo, lo capacita para tomar parte en el proceso de entendimiento y para afirmar su propia identidad. Es importante hacer notar que el interés técnico genera una racionalidad instrumental, equivalente a lo que Quinn (2009) llamaría las competencias instrumentales, soportadas en los roles de la gerencia, planificación, organización, dirección y control.

Asimismo, lo planteado por Haberman (2008) y Quinn (2009) evidencian que las competencias no pueden ser buscadas fuera de la acción gerencial que ignore los valores, normas, intereses, el pensamiento y la acción; en todo caso, el desconocimiento del contexto de interés en relación con el significado organizacional puede limitar la adquisición y desarrollo de competencias. En consecuencia, una competencia sugiere 


\section{CIENCIAMATRIA}

Revista Interdisciplinaria de Humanidades, Educación, Ciencia y Tecnología

Año VI. Vol. VI. N¹0. Enero - Julio 2020

Hecho el depósito de ley: pp201602FA4721

ISSN-L: 2542-3029; ISSN: 2610-802X

Universidad Nacional Experimental Francisco de Miranda (UNEFM). Santa Ana de Coro. Venezuela

Jorge Eduardo Jalil Ponce; Vicenta Elizabeth Pisco Sornoza; Johana María Zambrano Sornoza

tanto la posesión del conocimiento como la capacidad conductual para actuar adecuadamente. Para desarrollar las competencias el gerente debe tener acceso a los conocimientos y tener la oportunidad de practicar las destrezas. Sin embargo, cuando se ocupan puestos de liderazgo, como es el caso del gerente universitario, para ser operativo tiene que ser eficaz en todas las áreas de los valores competitivos, es decir, la conceptualización no es suficiente, tienen que ser capaces de actuar.

Al respecto, Quinn (2009) plantea: "la competencia significa poseer los conocimientos y las destrezas necesarias para desempeñar determinada tarea o rol"(p.17). Tal como se ha expuesto, la acción gerencial requiere de competencias las cuales pueden ser categorizadas de acuerdo a la temporalidad y pertinencia de la acción en decadente, dominante y emergente. Estas competencias se operacionalizan no sólo por los conocimientos, habilidades y destrezas que posea el gerente, sino también por un esquema de acción de trabajo, de valores en competencia que determinen la intención de la acción gerencial, en este caso académica.

De lo planteado se infiere que el éxito del gerente educativo depende, en gran medida, de las competencias que logre activar, no sólo en la rutina de los centros de educación superior sino en las circunstancias imprevistas. De allí, la importancia que el gerente se conciba a sí mismo como un ente corporativo, que asuma retos en la organización para atender los intereses y metas comunes. Bajo esta propuesta, el artículo tuvo como objetivo identificar las competencias instrumentales del gerente en el sistema de educación superior

\section{DESARROLLO}

Es imprescindible que el gerente universitario atienda sus funciones y distribuya responsabilidades para hacer posible los objetivos que persigue, apoyado en el liderazgo de grupo. En efecto, cuando los profesionales educativos ocupan puestos de liderazgo, su operatividad tiene que ser eficaz en todas las áreas de los valores competitivos. Es 


\section{CIENCIAMATRIA}

Revista Interdisciplinaria de Humanidades, Educación, Ciencia y Tecnología

Año VI. Vol. VI. N¹0. Enero - Julio 2020

Hecho el depósito de ley: pp201602FA4721

ISSN-L: 2542-3029; ISSN: 2610-802X

Universidad Nacional Experimental Francisco de Miranda (UNEFM). Santa Ana de Coro. Venezuela

Jorge Eduardo Jalil Ponce; Vicenta Elizabeth Pisco Sornoza; Johana María Zambrano Sornoza

decir, tienen que poseer las competencias instrumentales y valorativas que les permitan operar en cada una de las funciones que les toca cumplir, en este sentido, Barrientos Oradini, Araya Castillo, Veloso Salazar \& Herrera Ciudad (2019), siendo importante por parte de la gerencia educativa, fomentar competencias emocionales asertivas en los docentes con la finalidad de generar una mejor disposición al trabajo.

\section{Competencias Instrumentales del gerente}

Melinkoff (2001), plantea que el gerente educativo necesita tener competencias para planificar, organizar, dirigir y controlar; planificar implica determinar cursos de acción, la estructura y la pertinencia de los objetivos que orientan los logros de la organización. Las competencias del gerente educativo permiten sistematizar las acciones a través de una serie de procedimientos que posibilitan ir penetrando progresivamente en las competencias que su profesión le exige.

De allí, que corresponde al gerente de las instituciones universitarias cumplir eficientemente con sus competencias para poder lograr óptimos resultados con el menor esfuerzo. Es necesario que el gerente en su gestión aproveche las innovaciones técnicas y los valores que el nivel de Educación superior le demanda para acercarse a su función social.

En la Educación superior, se requiere un conjunto de competencias de parte del gerente, las cuales proporcionan una estructura que permite mantener a los centros educativos universitarios; cuando esto se efectúa debidamente favorece la gestión y misión institucional. En efecto, señala Melinkoff (2001), que entre las competencias instrumentales de la gerencia educativa se encuentran una serie de funciones que la definen como tal; entre éstas:

Competencia de Planificador: una de las funciones básicas que definen las competencias del gerente es la planificación, ella supone actividades como el diagnóstico 


\section{CIENCIAMATRIA}

Revista Interdisciplinaria de Humanidades, Educación, Ciencia y Tecnología

Año VI. Vol. VI. N¹0. Enero - Julio 2020

Hecho el depósito de ley: pp201602FA4721

ISSN-L: 2542-3029; ISSN: 2610-802X

Universidad Nacional Experimental Francisco de Miranda (UNEFM). Santa Ana de Coro. Venezuela

Jorge Eduardo Jalil Ponce; Vicenta Elizabeth Pisco Sornoza; Johana María Zambrano Sornoza

de las situaciones y la formulación de políticas, programas, objetivos, metas y estrategias. Al respecto Terry (2005) señala:

La planificación es una función primaria de la administración. Para que sobreviva cualquier organización es necesario cierto grado de planeación. Es el proceso por el cual no sólo se establecen los objetivos, sino que también se integran todos los recursos para lograrlos en forma

provechosa.(p.36)

De allí que la planificación permite determinar los recursos de la organización e integrarlos para relacionarlos en función de los objetivos previstos. En el mismo orden de ideas, Smith (2009) señala:

La planificación es pensar totalmente las acciones necesarias para que se alcance determinado objetivo o se cumpla una misión. Esto implica el empleo de juicio y discreción para determinar cuáles acciones serán más eficaces para obtener del futuro. Para planificar, la guía o norma para realizar con eficacia la misión de la unidad se recomiendan los pasos siguientes: examen del objetivo, finalidad o misión que debe realizarse, determinar qué hacer para lograr el objetivo, finalidad o misión, escoger la acción más eficaz y factible que pueda emprenderse.(p.29)

Competencia de Organizador: para que un centro educativo logre en forma óptima los objetivos que se propone, es necesario que se organice para el ordenamiento de los distintos elementos que intervienen en el proceso gerencial. Al respecto, Terry (2005) señala:

Organizar es el establecimiento de relaciones efectivas de comportamiento entre personas de manera que puedan trabajar juntas, con eficacia y puedan tener una satisfacción personal al hacer tareas seleccionadas bajo condiciones ambientales dadas para el propósito de alcanzar alguna meta $u$ objetivo.(p.304)

En efecto, el propósito fundamental de la organización en los centros de educación superior es que exista una coordinación de sus miembros, cuyas acciones aseguren de forma sistemática un modelo de esfuerzo colectivo donde ellos puedan actuar con 


\section{CIENCIAMATRIA}

Revista Interdisciplinaria de Humanidades, Educación, Ciencia y Tecnología

Año VI. Vol. VI. N¹0. Enero - Julio 2020

Hecho el depósito de ley: pp201602FA4721

ISSN-L: 2542-3029; ISSN: 2610-802X

Universidad Nacional Experimental Francisco de Miranda (UNEFM). Santa Ana de Coro. Venezuela

Jorge Eduardo Jalil Ponce; Vicenta Elizabeth Pisco Sornoza; Johana María Zambrano Sornoza

seguridad, estableciendo una función independiente de las personas o individuos que pertenecen a ella.

De allí que Bittel y Ramsey (2003), expresan que "una organización pueda concebirse como el conjunto de acciones coordinadas de dos o más personas con el propósito de llegar a un objetivo común"(p.43). Según los autores, la organización, si tiene buena arquitectura, todos sus componentes funcionan armoniosamente, en correspondencia a la misión y visión de ésta, pero dentro de ciertas exigencias colectivas y productivas. En esta perspectiva, Melinkoff (2001), afirma que "la organización es una función pre ejecutiva de la gerencia, que se complementa con la dirección, pues solamente a través de ese proceso de complementación es factible lograr el objetivo, que por sí misma no alcanzaría"(p.10). En efecto, la organización es el proceso de disponer y destinar el trabajo, la autoridad y los recursos entre los miembros de una organización de manera eficiente.

Por otro lado, Robbins (2010), señala que la organización "se encarga de definir qué actividades se realizarán, quién las llevará a cabo, cómo se agruparán, quién dependerá de quién y dónde se tomarán las decisiones"(p.5). El propósito fundamental de una organización es ayudar a crear un ambiente propio para la actividad humana, donde los gerentes son los responsables de que las organizaciones alcancen sus metas.

Competencia para Direccionar: la dirección consiste en motivar a los empleados para que desempeñen su actividad y así, lograr los objetivos de la organización, mediante el liderazgo y la toma de decisiones comunes. Al referirse a la dirección educativa, Terry (2005) señala: "la acción de dirección es un proceso que imprime orden y hace crecer la organización como sistema y se asocia con la planificación y organización"(p.38). La dirección así estudiada necesita prestar atención a la selección de método y herramientas gerenciales, considerando los factores que pueden limitarla, sin olvidar que cada persona 


\section{CIENCIAMATRIA}

Revista Interdisciplinaria de Humanidades, Educación, Ciencia y Tecnología

Año VI. Vol. VI. N¹0. Enero - Julio 2020

Hecho el depósito de ley: pp201602FA4721

ISSN-L: 2542-3029; ISSN: 2610-802X

Universidad Nacional Experimental Francisco de Miranda (UNEFM). Santa Ana de Coro. Venezuela

Jorge Eduardo Jalil Ponce; Vicenta Elizabeth Pisco Sornoza; Johana María Zambrano Sornoza

es una individualidad y desea que sus necesidades sean atendidas.Los autores Koontz y Weirich (2000) definen la dirección de la siguiente forma:

Consiste en influir en los seres humanos para que contribuyan a la obtención de las metas de la organización y del grupo; se refiere predominantemente a los aspectos interpersonales de la administración, todos los gerentes están de acuerdo en que sus problemas más importantes surgen de los seres humanos (sus deseos y actitudes, su conducta como individuos y en grupo) y que los gerentes eficaces también necesitan ser buenos líderes. (p.19)

Es responsabilidad de la gerencia es buscar la conciliación de los intereses de la organización a través de los objetivos previstos, para ello cuenta con los siguientes medios: la autoridad, la disciplina, el factor social o humano, la dirección y la responsabilidad. La dirección denota un proceso interpersonal, donde los gerentes se comunican con sus subalternos respecto a la ejecución del trabajo, facilitando a través del intercambio de información acerca de los problemas técnicos, de coordinación y de motivación con el fin de resolver conflictos organizacionales, siendo pertinente focalizar acciones desde lo sistémico, tal como proponen Aldana, Salón \& Guzmán (2019), con la finalidad de ejecutar diversas tareas desde el contexto organizacional.

En efecto, la dirección es la función fundamental a través de la cual se ejerce la función gerencial. Corresponde a ésta, la tarea de poner en funcionamiento el aparato administrativo cuya estructura ha sido integrada a través de la organización. Un buen gerente se identifica con los principios de la gerencia moderna, entre los cuales se destacan los de eficiencia, toma de decisiones, coordinación e integración. Por su parte, Chiavenato (2014), establecen que:

Los principios de la dirección son: 1) Efectividad para dirigir hacia los objetivos. Mientras más efectiva sea la forma de dirigir, mayor será la contribución de los subordinados para lograr los objetivos de la organización; 2) Armonía con los objetivos. Aunque los empleados trabajen por necesidad, éstos deben armonizar con los objetivos de la empresa; 3) Unidad de mando. Este principio es también de la etapa de la organización y refiere que los subordinados sean responsables ante su supervisor. 


\section{CIENCIAMATRIA}

Revista Interdisciplinaria de Humanidades, Educación, Ciencia y Tecnología

Año VI. Vol. VI. N¹0. Enero - Julio 2020

Hecho el depósito de ley: pp201602FA4721

ISSN-L: 2542-3029; ISSN: 2610-802X

Universidad Nacional Experimental Francisco de Miranda (UNEFM). Santa Ana de Coro. Venezuela

Jorge Eduardo Jalil Ponce; Vicenta Elizabeth Pisco Sornoza; Johana María Zambrano Sornoza

Señalan los autores que para lograr una buena dirección administrativa los gerentes deben: procurar que las personas se sientan importantes, reconocer las diferencias individuales, orientar a los empleados, practicar la administración participativa, saber escuchar, evitar discusiones, emplear preguntas para persuadir y proporcionar supervisión efectiva. En consecuencia, reconocer las diferencias individuales y hacer que las personas se sientan importantes es esencial en la gerencia, por cuanto el recurso humano es el elemento clave de la productividad, siendo indispensable generar una gerencia transformadora como indican Morgado, Aldana Zavala \& Isea Argüelles (2019). En tal sentido, Robbins (2010), señala que "dirigir personas involucra los conceptos de motivación, liderazgo, guía, estímulo y actuación, lo que establece claramente la relación entre la dirección y el factor humano de la organización, se debe considerar al personal como el recurso humano más importante de la empresa" (p.98). Por tanto, el factor humano debe ser motivado a fin de que se sienta comprometido en la institución para su mejor desenvolvimiento en la misma. De allí que el logro de los objetivos depende de la eficiencia con que el grupo sea dirigido.

Competencias de Controlador: el control es un proceso que ayuda a los gerentes a realizar el seguimiento de la eficacia de su planeación, su organización y su dirección; la realización de informes, comparaciones y evaluaciones del avance constituye el fundamento para intervenir, hacer ajustes, replanear y aplicar medidas correctivas cuando se necesitan. Smith (2009) en cuanto al control considera:

Es difícil alcanzar la perfección, se hace necesario poner en vigor controles que impidan que se produzcan errores, o para descubrir lo que funciona mal y ponerle remedio. Para llevar a cabo esto, el supervisor tiene que mantener una vigilancia estrecha de todo cuanto sucede. El control adecuado depende de una corriente de información significativa, precisa y oportuna que corra de arriba abajo y de un lado a otro de la organización. (p.42) 


\section{CIENCIAMATRIA}

Revista Interdisciplinaria de Humanidades, Educación, Ciencia y Tecnología

Año VI. Vol. VI. N¹0. Enero - Julio 2020

Hecho el depósito de ley: pp201602FA4721

ISSN-L: 2542-3029; ISSN: 2610-802X

Universidad Nacional Experimental Francisco de Miranda (UNEFM). Santa Ana de Coro. Venezuela

Jorge Eduardo Jalil Ponce; Vicenta Elizabeth Pisco Sornoza; Johana María Zambrano Sornoza

El control es un proceso que atiende la indagación, la confrontación, la delegación y la toma de decisiones. Se sustenta en un desempeño logrado y otro deseado y permite delinear cursos de acción, con fines de equilibrio institucional.

Al respecto, Newman (2004), define el control como "la fase final de la dirección, en la cual se asegura que la ejecución se realice de acuerdo a los pasos previamente concertados" (p.45). Según el autor, la función controladora está basada en un control que no es de tipo vertical y autoritario, sino una fase cooperativa de intercambio de informaciones y opiniones, los objetivos aparecen formulados y la labor entre los miembros de la organización se realiza de manera coordinada para el logro de objetivos comunes. El control es un proceso indispensable en todas las etapas administrativas y a todos los niveles jerárquicos.

\section{METODOLOGÍA}

Para dar respuesta al objetivo de investigación se siguió una metodología de tipo descriptiva con diseño de campo, no experimental y transeccional. Es descriptiva por cuanto se describieron los hechos tal y como ocurrieron en la realidad sustentándose en lo planteado por Hurtado (2011) quien afirma: "los estudios descriptivos infieren la descripción acerca de las singularidades de una realidad estudiada ", por lo tanto, en este caso se describieron las competencias instrumentales gerenciales, que se manifiestan en el nivel de educación superior. En relación con la tipología de campo, el estudio se ajustó a esta modalidad por cuanto se apoyó en lo expuesto por Hernández y col (2014), quienes afirman que en este tipo de estudio se recolecta la información fundamentándose en testificaciones basadas en la realidad. Por lo tanto en este caso se recolectaron datos directamente de la realidad donde se evaluó el problema, sin manipular las variables. La técnica de investigación aplicada fue la encuesta medida a través de un cuestionario como instrumento de recolección de datos. Éste estuvo compuesto por 12 ítems con opciones de respuesta politómica que fue aplicado a los docentes con cargos gerenciales universitarios. 
CIENCIAMATRIA

Revista Interdisciplinaria de Humanidades, Educación, Ciencia y Tecnología

Año VI. Vol. VI. N¹0. Enero - Julio 2020

Hecho el depósito de ley: pp201602FA4721

ISSN-L: 2542-3029; ISSN: 2610-802X

Universidad Nacional Experimental Francisco de Miranda (UNEFM). Santa Ana de Coro. Venezuela

Jorge Eduardo Jalil Ponce; Vicenta Elizabeth Pisco Sornoza; Johana María Zambrano Sornoza

\section{RESULTADOS}

Los resultados del estudio, permitieron responder a la interrogante: ¿Cómo serán las competencias instrumentales del gerente en el sistema de educación superior?

El análisis, se desarrolló, mediante la interpretación de todas las respuestas obtenidas del cuestionario. Asimismo, se expresa la opinión del investigador con relación a las teorías que se indagaron y propusieron en el estudio. Es relevante destacar, que dicho análisis y discusión de los resultados se realizó para dar respuesta al objetivo propuesto

\section{Tabla 1}

Análisis descriptivo del indicador Competencias instrumentales del gerente como Planificador

\begin{tabular}{ccccc} 
& $\boldsymbol{F} \boldsymbol{R}$ & $\%$ & $\boldsymbol{F R A}$ & $\% \boldsymbol{A}$ \\
\hline Totalmente de acuerdo & 8 & 13 & 8 & 13 \\
De acuerdo & 12 & 20 & 20 & 33 \\
Ni de acuerdo ni en desacuerdo & 24 & 40 & 44 & 73 \\
En desacuerdo & 12 & 20 & 56 & 93 \\
Totalmente en desacuerdo & 4 & 7 & 60 & 100 \\
MEDIA & \multicolumn{5}{c}{$\mathbf{3 , 8 2}$} \\
DESVIACION ESTANDAR & \multicolumn{5}{c}{$\mathbf{0 , 2 1}$} \\
MEDIANA & $\mathbf{2 , 5 5}$
\end{tabular}

Fuente: Elaboración propia (2019)

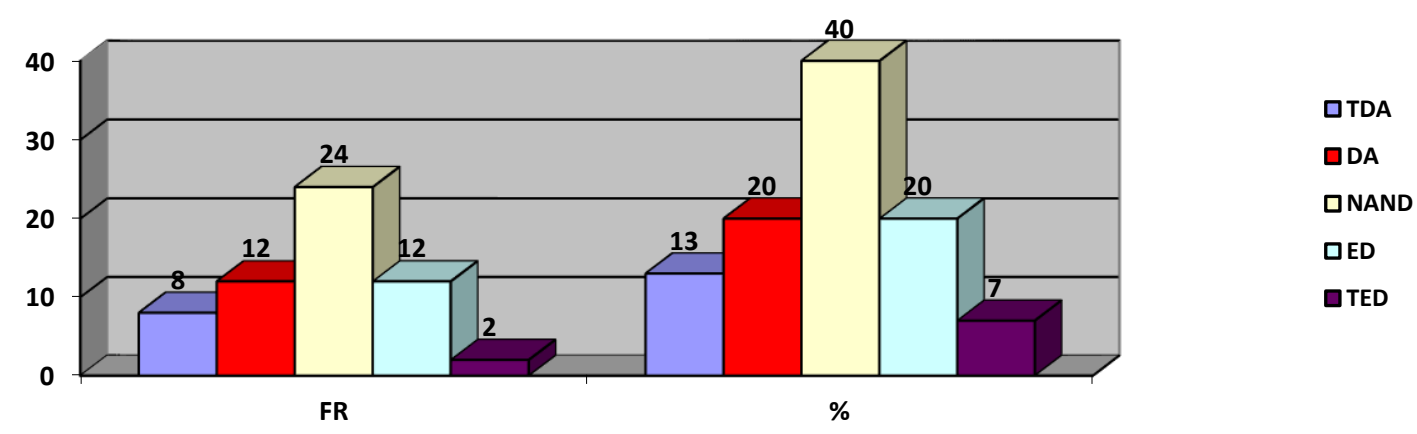

\section{Gráfico 1}

Análisis descriptivo del indicador Competencias instrumentales del gerente como planificador. Fuente: Elaboración propia (2019) 


\section{CIENCIAMATRIA}

Revista Interdisciplinaria de Humanidades, Educación, Ciencia y Tecnología

Año VI. Vol. VI. N¹0. Enero - Julio 2020

Hecho el depósito de ley: pp201602FA4721

ISSN-L: 2542-3029; ISSN: 2610-802X

Universidad Nacional Experimental Francisco de Miranda (UNEFM). Santa Ana de Coro. Venezuela

Jorge Eduardo Jalil Ponce; Vicenta Elizabeth Pisco Sornoza; Johana María Zambrano Sornoza

En la tabla y gráfico 1, se presenta el análisis descriptivo del indicador competencias instrumentales del gerente universitario como planificador en la que se observa, que el $40 \%$ del total de los encuestados coincidieron en opinar que ni estaban de acuerdo ni en desacuerdo en que: en la institución se establecen los objetivos en atención a los recursos que se disponen para lograrlos en forma provechosa, se organizan e integran para relacionarlos en función de los objetivos previstos y se emplea el juicio y la discreción para determinar cuáles acciones serán más eficaces para lograr lo planificado institucionalmente. Seguido del $20 \%$ por igual que consideraron estar en desacuerdo y de acuerdo. Los menores porcentajes se indicaron para las alternativas totalmente de acuerdo y totalmente en desacuerdo con el $13 \%$ y $7 \%$ respectivamente. Asimismo, se observa que el valor de la media fue de 3,32 con una desviación estándar de $\pm 0,21$, evaluándose como de moderada eficiencia, implicando que en opinión de los investigados la misma puede variar entre las alternativas en desacuerdo y de acuerdo. La mediana con un valor 2,55 se ubicó por debajo de la media señalando que existe una tendencia de opinión hacia las alternativas bajas de medición.

Al respecto, cabe destacar que Terry (2005) señala, que la planificación es una función primaria de la administración. Para que sobreviva cualquier organización es necesario cierto grado de planeación. Es el proceso por el cual no sólo se establecen los objetivos, sino que también se integran todos los recursos para lograrlos en forma provechosa. 
CIENCIAMATRIA

Revista Interdisciplinaria de Humanidades, Educación, Ciencia y Tecnología

Año VI. Vol. VI. N¹0. Enero - Julio 2020

Hecho el depósito de ley: pp201602FA4721

ISSN-L: 2542-3029; ISSN: 2610-802X

Universidad Nacional Experimental Francisco de Miranda (UNEFM). Santa Ana de Coro. Venezuela

Jorge Eduardo Jalil Ponce; Vicenta Elizabeth Pisco Sornoza; Johana María Zambrano Sornoza

\section{Tabla 2}

Análisis Descriptivo del Indicador Competencias instrumentales del gerente universitario como Organizador.

\begin{tabular}{|c|c|c|c|c|}
\hline ANÁLISIS/ CRITERIOS & $\boldsymbol{F} \boldsymbol{R}$ & $\%$ & FRA & $\% A$ \\
\hline Totalmente de acuerdo & 6 & 10 & 6 & 10 \\
\hline De acuerdo & 30 & 50 & 36 & 60 \\
\hline $\begin{array}{c}\text { Ni de acuerdo ni en } \\
\text { desacuerdo }\end{array}$ & 14 & 23 & 50 & 83 \\
\hline En desacuerdo & 10 & 17 & 60 & 100 \\
\hline Totalmente en desacuerdo & 0 & 0 & 60 & 100 \\
\hline MEDIA & \multicolumn{4}{|c|}{2,62} \\
\hline DESVIACION ESTANDAR & \multicolumn{4}{|c|}{0,11} \\
\hline MEDIANA & \multicolumn{4}{|c|}{2,35} \\
\hline
\end{tabular}

Fuente: Elaboración propia (2019)

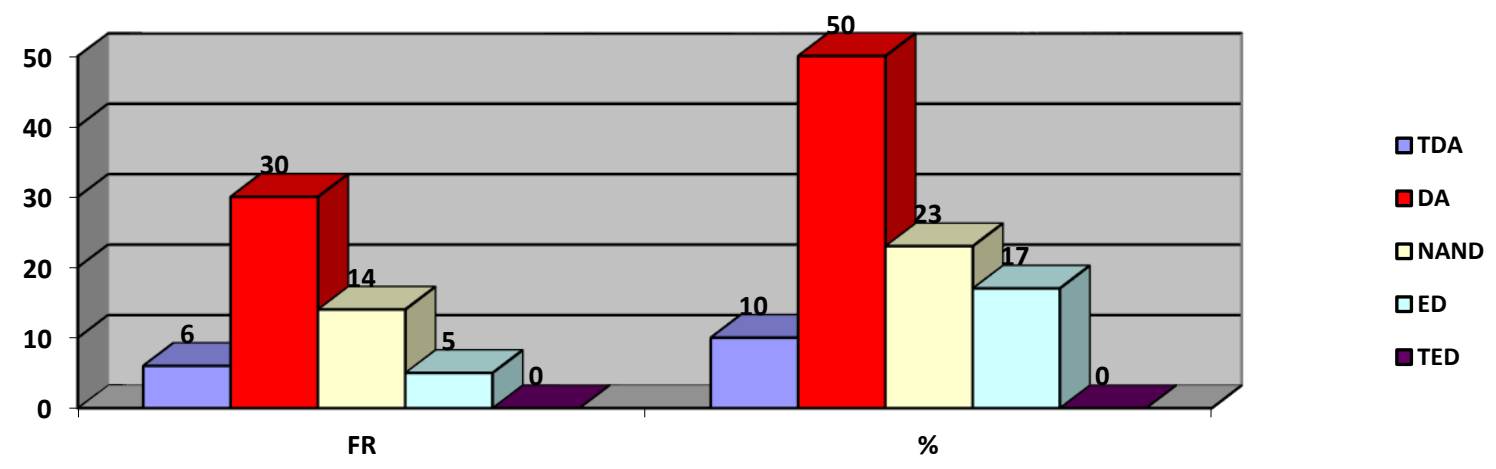

Gráfico 2. Análisis descriptivo del indicador competencias instrumentales del gerente universitario como organizador.

Fuente: Elaboración propia (2019)

En la tabla y gráfico 2, se presenta el análisis descriptivo del indicador competencias instrumentales del gerente universitario como organizador, en la que se indica que el $50 \%$ del total de los encuestados coincidieron en opinar en que estaban de acuerdo en que es posible trabajar en equipo para lograr eficacia y satisfacción personal al hacer tareas seleccionadas y concebir acciones coordinadas de dos o más personas con el propósito 


\section{CIENCIAMATRIA}

Revista Interdisciplinaria de Humanidades, Educación, Ciencia y Tecnología

Año VI. Vol. VI. N¹0. Enero - Julio 2020

Hecho el depósito de ley: pp201602FA4721

ISSN-L: 2542-3029; ISSN: 2610-802X

Universidad Nacional Experimental Francisco de Miranda (UNEFM). Santa Ana de Coro. Venezuela

Jorge Eduardo Jalil Ponce; Vicenta Elizabeth Pisco Sornoza; Johana María Zambrano Sornoza

de llegar a un objetivo común así como desde la institución trabajar complementando sus acciones con la dirección. Seguido del $23 \%$ y $17 \%$ que consideraron estar ni de acuerdo ni en desacuerdo y en desacuerdo.

Los menores porcentajes se indicaron para las alternativas extremas de totalmente de acuerdo y totalmente en desacuerdo con el $10 \%$ y $0 \%$ del total de las opiniones. De igual manera se observa que el valor de la media fue de 3,62 con una desviación estándar de $\pm 0,11$, evaluándose como eficiente entre los investigados señalando que la misma puede variar entre las alternativas ni de acuerdo ni en desacuerdo y en desacuerdo, evaluando como medianamente favorable la competencia instrumental de organización. La mediana con un valor 2,35 se ubicó por debajo de las medias señalando que existe una tendencia hacia la alternativa baja de totalmente en desacuerdo.

Desde esta perspectiva, Melinkoff (2001), afirma que la organización es una función pre ejecutiva de la gerencia, que se complementa con la dirección, pues solamente a través de ese proceso de complementación es factible lograr el objetivo, que por sí misma no alcanzaría. En efecto, la organización es el proceso de disponer y destinar el trabajo, la autoridad y los recursos entre los miembros de una organización de manera eficiente.

Tabla 3. Análisis Descriptivo del Indicador Competencias instrumentales del gerente universitario para direccionar.

\begin{tabular}{|c|c|c|c|c|}
\hline ANÁLISIS/ CRITERIOS & $F \boldsymbol{R}$ & $\%$ & FRA & $\% A$ \\
\hline Totalmente de acuerdo & 6 & 10 & 6 & 10 \\
\hline De acuerdo & 30 & 50 & 36 & 60 \\
\hline $\begin{array}{c}\text { Ni de acuerdo ni en } \\
\text { desacuerdo }\end{array}$ & 12 & 20 & 48 & 80 \\
\hline En desacuerdo & 8 & 13 & 56 & 93 \\
\hline Totalmente en desacuerdo & 4 & 7 & 60 & 100 \\
\hline $\begin{array}{c}\text { MEDIA } \\
\text { DESVIACION ESTANDAR } \\
\text { MEDIANA }\end{array}$ & \multicolumn{4}{|c|}{$\begin{array}{l}3,89 \\
0,08 \\
3,22\end{array}$} \\
\hline
\end{tabular}

Fuente: Elaboración propia (2019) 


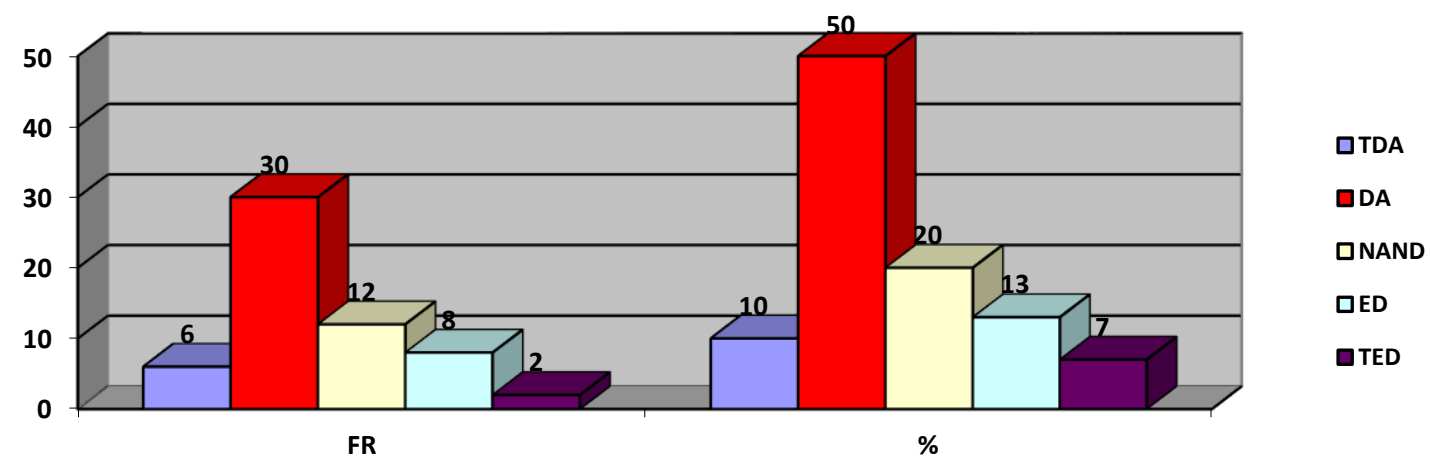

Gráfico 3. Análisis Descriptivo del Indicador Competencias Instrumentales del gerente universitario para direccionar.

Fuente: Elaboración propia (2019)

En la tabla y gráfico 3, se presenta el análisis descriptivo del indicador competencias instrumentales del gerente universitario para direccionar, en la que se observa que el $50 \%$ del total de los encuestados coincidieron en opinar en que estaban de acuerdo en que: el personal es motivado para que desempeñen su actividad y así, lograr los objetivos de la institución, los gerentes universitarios influyen en el personal para que contribuyan a la obtención de las meta de la organización y del grupo y exista armonía entre el personal de los diferentes departamentos que se rigen en los recintos universitarios que permiten efectividad para cumplir con los objetivos propuestos.

Seguido del $20 \%$ y $13 \%$ que consideraron estar ni de acuerdo ni en desacuerdo y en desacuerdo. Los menores porcentajes se indicaron para las alternativas totalmente de acuerdo y totalmente en desacuerdo con el $10 \%$ y $7 \%$ del total de las opiniones. Asimismo, se observa que el valor de la media fue de 3,89 con una desviación estándar de $\pm 0,08$, evaluándose como eficiente entre los investigados señalando que la misma puede variar entre las alternativas ni de acuerdo ni en desacuerdo y totalmente de acuerdo, evaluando como favorable la competencia instrumental de dirección. La mediana con un valor 3.22 se ubicó por debajo de las medias señalando que existe una tendencia hacia las alternativas bajas de medición. 


\section{CIENCIAMATRIA}

Revista Interdisciplinaria de Humanidades, Educación, Ciencia y Tecnología

Año VI. Vol. VI. N¹0. Enero - Julio 2020

Hecho el depósito de ley: pp201602FA4721

ISSN-L: 2542-3029; ISSN: 2610-802X

Universidad Nacional Experimental Francisco de Miranda (UNEFM). Santa Ana de Coro. Venezuela

Jorge Eduardo Jalil Ponce; Vicenta Elizabeth Pisco Sornoza; Johana María Zambrano Sornoza

Lo expuesto, difiere con lo propuesto por Chiavenato (2004), quien señala que los principios de la dirección han de estar enfocados en la efectividad para dirigir hacia los objetivos. Mientras más efectiva sea la forma de dirigir, mayor será la contribución de los subordinados para lograr los objetivos de la organización, también en armonía con los objetivos institucionales. Aunque los empleados trabajen por necesidad, éstos deben armonizar con los objetivos de la misma. De allí que para lograr una buena dirección administrativa el personal debe procurar que los personas se sientan importantes, reconocer las diferencias individuales, orientar a los empleados, practicar la administración participativa, saber escuchar, evitar discusiones, emplear preguntas para persuadir y proporcionar supervisión efectiva.

Tabla 4. Análisis Descriptivo del Indicador de las Competencias instrumentales del gerente como Controlador.

\begin{tabular}{ccccc} 
ANÁLISIS/ CRITERIOS & $\boldsymbol{F R}$ & $\%$ & $\boldsymbol{F R A}$ & $\% \boldsymbol{A}$ \\
\hline Totalmente de acuerdo & 2 & 3 & 2 & 3 \\
De acuerdo & 18 & 30 & 20 & 33 \\
Ni de acuerdo ni en desacuerdo & 26 & 43 & 46 & 76 \\
En desacuerdo & 10 & 17 & 56 & 93 \\
Totalmente en desacuerdo & 4 & 7 & 60 & 100 \\
MEDIA & \multicolumn{5}{c}{$\mathbf{3 , 9 0}$} \\
DESVIACION ESTANDAR & \multicolumn{5}{c}{$\mathbf{0 , 1 2}$} \\
MEDIANA & $\mathbf{3 , 9 5}$
\end{tabular}

Fuente: Elaboración propia (2019)

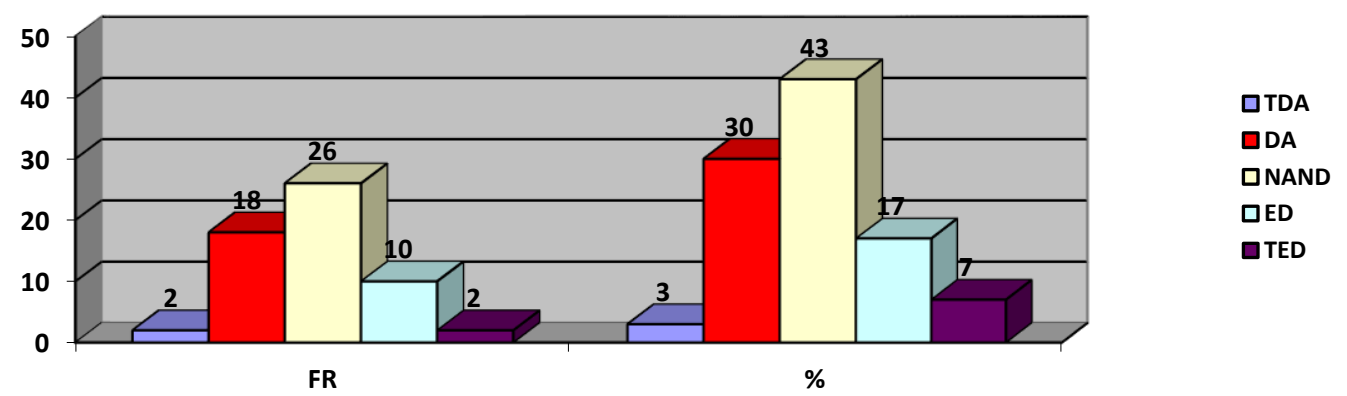

Gráfico 4: Análisis Descriptivo del Indicador Competencias instrumentales del gerente como Controlador.

Fuente: Elaboración propia (2019) 


\section{CIENCIAMATRIA}

Revista Interdisciplinaria de Humanidades, Educación, Ciencia y Tecnología

Año VI. Vol. VI. N¹0. Enero - Julio 2020

Hecho el depósito de ley: pp201602FA4721

ISSN-L: 2542-3029; ISSN: 2610-802X

Universidad Nacional Experimental Francisco de Miranda (UNEFM). Santa Ana de Coro. Venezuela

Jorge Eduardo Jalil Ponce; Vicenta Elizabeth Pisco Sornoza; Johana María Zambrano Sornoza

En la tabla y gráfico 4, se presenta el análisis descriptivo del indicador competencias instrumentales del gerente universitario como controlador, en la que se observa, que el $43 \%$ del total de los encuestados coincidieron en opinar en un ni de acuerdo ni en desacuerdo, en qué; se hace seguimiento de la eficacia de lo planeado a través de la realización de informes, comparaciones y evaluaciones del avance de las tareas a cumplir, se maneja la información significativa, precisa y oportuna de los diferente niveles jerárquicos para llevar a cabo sus acciones laborales y se controla la ejecución de las acciones laborales de acuerdo a los pasos previamente concertados. Seguido del $30 \%$ y $17 \%$ que consideraron estar de acuerdo y en desacuerdo.

Los menores porcentajes se indicaron para las alternativas extremas de totalmente de acuerdo y totalmente en desacuerdo con el 3\% y $7 \%$ respectivamente del total de las opiniones. Asimismo, se observa que el valor de la media fue de 2,90 con una desviación estándar de $\pm 0,12$, evaluándose como moderadamente eficiente entre los investigados señalando que la misma puede variar entre las alternativas de acuerdo y en desacuerdo. La mediana con un valor 3.95 se ubicó por debajo de las medias señalando que existe una tendencia hacia las alternativas bajas de medición.

Es de destacar, que estos resultados difieren de la posición de Smith (2009) en cuanto que este autor considera que se hace necesario poner en vigor controles que impidan que se produzcan errores, o para descubrir lo que funciona mal y ponerle remedio. Para llevar a cabo esto, los gerentes tienen que mantener una vigilancia estrecha de todo cuanto sucede. El control adecuado depende de una corriente de información significativa, precisa y oportuna que corra de arriba abajo y de un lado a otro de la institución.

\section{CONCLUSIONES}

En cuanto a las competencias instrumentales que aplican los gerentes universitarios, se indico un nivel eficiente con el cumplimiento de las mismas, constatándose un nivel medianamente eficiente en la cual los espacios universitarios establecen los 
CIENCIAMATRIA

Revista Interdisciplinaria de Humanidades, Educación, Ciencia y Tecnología

Año VI. Vol. VI. N¹0. Enero - Julio 2020

Hecho el depósito de ley: pp201602FA4721

ISSN-L: 2542-3029; ISSN: 2610-802X

Universidad Nacional Experimental Francisco de Miranda (UNEFM). Santa Ana de Coro. Venezuela

Jorge Eduardo Jalil Ponce; Vicenta Elizabeth Pisco Sornoza; Johana María Zambrano Sornoza

objetivos a cumplir en relación con los recursos materiales, pedagógicos y humanos que dispongan.

Existe entre los investigados un ni acuerdo ni en desacuerdo con la competencia instrumental del gerente universitario como planificador, a pesar de ser una función primaria de todo gerente, alejando las posibilidades de aprovechar los recursos de los cuales se disponen para llevar a cabo la planificación de los objetivos institucionales.

Existe acuerdo, en opinión de la mitad de los investigados, relacionado con las competencias instrumentales del gerente universitario como organizador, indicando que es posible trabajar en equipo para lograr eficacia y satisfacción personal al hacer tareas seleccionadas y concebir acciones coordinadas de dos o más docentes con el propósito de llegar a un objetivo común así como desde la institución trabajar complementando sus acciones con la dirección.

Se constató el estar de acuerdo, desde una proporción de igualdad de opiniones, que las competencias instrumentales del gerente universitario para direccionar al personal para motivarlos a ejercer el desempeño de sus actividades en pro de lograr los objetivos propuestos desde la gerencia universitaria.

En cuanto a las competencias instrumentales del gerente universitario como controlador, existe un ni de acuerdo ni en desacuerdo, en qué; se hace seguimiento con los vigores requeridos para controlar los posibles errores, o para descubrir lo que funciona mal y ponerle remedio. 
CIENCIAMATRIA

Revista Interdisciplinaria de Humanidades, Educación, Ciencia y Tecnología

Año VI. Vol. VI. N¹0. Enero - Julio 2020

Hecho el depósito de ley: pp201602FA4721

ISSN-L: 2542-3029; ISSN: 2610-802X

Universidad Nacional Experimental Francisco de Miranda (UNEFM). Santa Ana de Coro. Venezuela

Jorge Eduardo Jalil Ponce; Vicenta Elizabeth Pisco Sornoza; Johana María Zambrano Sornoza

\section{REFERENCIAS CONSULTADAS}

1. Aldana, J., Salón, M., \& Guzmán, N. (2019). Liderazgo sistémico en las competencias gerenciales docentes universitarias. CIENCIAMATRIA, 5(8), 50-74. Recuperado a partir de http://cienciamatriarevista.org.ve/index.php/cm/article/view/87

2. Barrientos Oradini, N., Araya Castillo, L., Veloso Salazar, V., \& Herrera Ciudad, C. (2019). Optimismo disposicional en docentes de universidades privadas en región metropolitana en Chile. Revista Arbitrada Interdisciplinaria Koinonía, 4(7), 168184. doi:http://dx.doi.org/10.35381/r.k.v4i7.199

3. Bittel y Ramsey (2003). Enciclopedia del Management. Editorial McGraw-Hill. México.

4. Chiavenato, I. (2004). Administración de los Recursos Humanos. McGraw-Hill. Bogotá.

5. Chiavenato , I. (2014). Introducción a la Teoría General de la Administración. 8va. Edición. McGraw Hill

6. Habermas, J. (2008). El futuro de la Naturaleza humana ¿ Hacia una eugenesia liberal? Editorial Paidós. Barcelona.

7. Hurtado, Y (2008). El proyecto de investigación. Metodología de la investigación Holística. Ediciones Quirón Sypal. Bogotá. Colombia.

8. Koontz, H. y Weihrich, H. (2000). Administración. Una Perspectiva Global. Décima Primera Edición. México: Editorial McGraw-Hill Interamericana S.A. de C.V.

9. Melinkoff. R. (2001). Los procesos administrativos. Bogotá. Editorial Panapo.

10. Morgado, C., Aldana Zavala, J., \& Isea Argüelles, J. (2019). Gerencia transformacional desde el accionar docente directivo para el abordaje del Contexto Escolar. CIENCIAMATRIA, 5(9), 48-72. https://doi.org/10.35381/cm.v5i9.99

11. Newman, A. (2004). Programación organización y control. Editorial Deustro Bilbao - Euzkadix

12. Quinn y Mintzberg. (2009). La planificación estratégica. Tomo II. Editorial prentice hall. México. 
CIENCIAMATRIA

Revista Interdisciplinaria de Humanidades, Educación, Ciencia y Tecnología

Año VI. Vol. VI. N¹0. Enero - Julio 2020

Hecho el depósito de ley: pp201602FA4721

ISSN-L: 2542-3029; ISSN: 2610-802X

Universidad Nacional Experimental Francisco de Miranda (UNEFM). Santa Ana de Coro. Venezuela

Jorge Eduardo Jalil Ponce; Vicenta Elizabeth Pisco Sornoza; Johana María Zambrano Sornoza

13. Robbins, Stepen (2010) La administración en el mundo de hoy. 5ª Edición. México. Editorial Prentice Hall.

14. Smith, C. (2009). Guías para Supervisores. Editorial Trillas. México.

15. Terry, George. (2005). Las competencias de un gerente. Editorial: Deusto, s. a México

\section{REFERENCES CONSULTED}

1. Aldana, J., Salón, M., \& Guzmán, N. (2019). Systemic leadership in university teaching management skills. SCIENCE, 5 (8), 50-74. Recovered from http://cienciamatriarevista.org.ve/index.php/cm/article/view/87

2. Barrientos Oradini, N., Araya Castillo, L., Veloso Salazar, V., \& Herrera Ciudad, C. (2019). Dispositional optimism in teachers of private universities in the metropolitan region in Chile. Interdisciplinary Arbitrated Review Koinonía, 4 (7), 168-184. doi: http: //dx.doi.org/10.35381/r.k.v4i7.199

3. Bittel and Ramsey (2003). Encyclopedia of Management. McGraw-Hill editorial. Mexico.

4. Chiavenato, I. (2004). Human Resources Administration. McGraw-Hill Bogota

5. Chiavenato, I. (2014). Introduction to the General Theory of Administration. 8th. Edition. McGraw Hill

6. Habermas, J. (2008). The future of human Nature Towards a liberal eugenics? Editorial Paidós. Barcelona.

7. Hurtado, Y (2008). The research project Holistic research methodology. Quirón Sypal editions. Bogota Colombia.

8. Koontz, H. and Weihrich, H. (2000). Administration. A Global Perspective Tenth First Edition. Mexico: Editorial McGraw-Hill Interamericana S.A. from C.V.

9. Melinkoff R. (2001). The administrative processes Bogota Panapo editorial. 


\section{CIENCIAMATRIA}

Revista Interdisciplinaria de Humanidades, Educación, Ciencia y Tecnología

Año VI. Vol. VI. N¹0. Enero - Julio 2020

Hecho el depósito de ley: pp201602FA4721

ISSN-L: 2542-3029; ISSN: 2610-802X

Universidad Nacional Experimental Francisco de Miranda (UNEFM). Santa Ana de Coro. Venezuela

Jorge Eduardo Jalil Ponce; Vicenta Elizabeth Pisco Sornoza; Johana María Zambrano Sornoza

10. Morgado, C., Aldana Zavala, J., \& Isea Argüelles, J. (2019). Transformational management from the directive teaching actions to address the School Context. SCIENCE, 5 (9), 48-72. https://doi.org/10.35381/cm.v5i9.99

11. Newman, A. (2004). Organization and control programming. Editorial Deustro Bilbao - Euzkadix

12. Quinn and Mintzberg. (2009). Strategic planning Volume II Editorial prentice hall. Mexico.

13. Robbins, Stepen (2010) Administration in the world today. 5th Edition. Mexico. Editorial Prentice Hall.

14. Smith, C. (2009). Guides for Supervisors. Editorial Trillas. Mexico.

15. Terry, George. (2005). The skills of a manager. Publisher: Deusto, s. to Mexico

(C2020 por los autores. Este artículo es de acceso abierto y distribuido según los términos y condiciones de la licencia Creative Commons Atribución-NoComercial-Compartirlgual 4.0 Internacional (CC BY-NC-SA 4.0) (https://creativecommons.org/licenses/by-nc-sa/4.0/). 